\title{
Spatially explicit individual-based modelling of insect- plant interactions: effects of level of detail in Queensland fruit fly models
}

\author{
Ming Wang a , Bronwen Cribb ${ }^{\text {b c }}$, Inigo Auzmendi a and Jim Hanan a \\ ${ }^{a}$ Queensland Alliance for Agriculture and Food Innovation (QAAFI), The University of Queensland, \\ Brisbane, QLD, Australia 4072 \\ ${ }^{b}$ Centre for Microscopy and Microanalysis, The University of Queensland, Brisbane, QLD, Australia 4072 \\ ${ }^{c}$ School of Biological Sciences, The University of Queensland, Brisbane, QLD, Australia 4072 \\ Email:m.wang@uq.edu.au
}

\begin{abstract}
A form of agent-based modelling known as individual-based modelling has been used widely to simulate hypothesised mechanisms underlying processes in the real world, especially in the field of behavioural ecology. To apply this approach to insect-plant interactions the Queensland fruit fly (Qfly), Bactrocera tryoni (Froggatt) (Diptera: Tephritidae), a major native insect pest of horticulture in Australia, will be examined. Due to restriction on use of some chemicals, currently there is no suitable management system for control of Qfly. However, better Qfly management can be potentially advanced by targeted behavioural, physiological and ecological research on this species. Many control options depend on behavioural fundamentals of Qfly such as foraging and movement patterns (e.g. trapping control technique), and the use of resources like protein and food sources (e.g. bait spray control technique).

In the present study, two 3-dimensional individual-based models with different levels of detail have been developed to simulate Qfly behaviour and movement patterns on host plants, one with NetLogo, and the other with L-studio. Both provide a dynamic platform for simulating insect movement decisions underlying searching and feeding behaviour. The spatial unit in the NetLogo model is based on 'vegetation cubes', while the Lindenmayer System (L-System) formalism-based approach underlying L-studio allows the model to have more detailed plant architecture, with individual leaves and stem segments. Both models predict that Qflies spend more time in the mid to upper canopy, which shows a good agreement with published literature. The overall objective is to test whether it is possible to predict fruit fly movement and distribution based on hypothesized behaviour and also assess whether such a model can provide insights that may have been missed in past experimental studies, investigate what level of detail is best used for addressing different types of questions from entomologists, and to show the advantages and disadvantages of both models.
\end{abstract}

Our study suggests that, the NetLogo model can be better used to investigate scientific questions like insect spatial population distribution on plant canopies and how different tree architectures affect their behaviour, while the L-system model is better to use to look at how foliage density and foliage position affect fruit flies behaviour and to simulate landscape scales such as orchards including multiple trees. Using the model with the right level of detail to inform, develop and test research allows new insights into insect-plant interactions and can inform experiments carried out in the field that have application in better pest management.

Keywords: Agent-based model, computational model, computer simulation, behavioural ecology, insectplant interactions 
Wang et al., Spatially explicit individual-based modelling of insect-plant interactions: effects of level of detail in Queensland fruit fly models

\section{INTRODUCTION}

Computational modelling is playing an increasingly significant role in understanding complex biological behaviours (Fisher and Henzinger, 2007). Biological scientists are finding that computational models, used to simulate hypothesised mechanisms underlying processes in the real world, can be of great value in understanding the systems they study (Di Ventura et al., 2006). In biology or ecology, computer simulations can create a virtual environment (in silico) and provide an alternative or a valuable supplement to in vitro or in vivo experiments (Hanan et al., 2002; Karr et al., 2012; Martin et al., 2013) with great potential for scientists to benefit if they consider using such models to inform, develop and test their research (Peck, 2004). Agent-based models that use simple rules to simulate individual behaviour, the interactions among and between individuals, and the interactions between individuals and their abiotic environment are known as individual-based models or IBM (Grimm and Railsback, 2005) in behavioural ecology and have been applied widely (Dyer et al., 2014; Guttal et al., 2012; Pe'er et al., 2013; Zurell et al., 2014). To explore the capacity of computational modelling to generate new perspectives in insect-plant interactions, the Queensland fruit fly (Qfly), Bactrocera tryoni (Froggatt) (Diptera: Tephritidae), with its host plants, provides a suitable target system for modelling. Because Qfly is a major insect pest of horticulture in Australia, and management is currently in flux (Clarke et al., 2011), development of such a model is timely. Additional insight into the behaviour of this pest could translate into applied outcomes.

Clarke et al. (2011) claim that better Qfly management can be advanced by targeted behavioural, physiological and ecological research on this species. Current options are based on the fundamental behaviours of Qfly such as foraging, movement patterns (e.g. trapping control technique), and the use of resources like protein and food sources (e.g. bait spray control technique) (Balagawi et al., 2012; Clarke et al., 2011). According to Clarke et al. (2011), trapping (lure and kill techniques) could become a more important management strategy now that some chemicals are restricted. However, the application of such a technique requires a better understanding of the way Qfly forage in plant canopies, because traps need to be placed at a proper position within or around tree canopies in order to maximise catch efficiency (Balagawi et al., 2012). A study conducted by Raghu et al. (2004) showed that host plant structure and microclimate can have significant effects on the abundance and behaviour of a related species, wild tobacco fruit fly, Bactrocera cacuminata (Hering) (Diptera: Tephritidae). For Qfly, Balagawi et al. (2012) and Balagawi et al. (2014) argued that traits of plant architecture can influence insect interactions with their host plants: most Qflies have been caught in the mid to upper canopy on fruiting plants. Several other species of tephritid, the apple maggot fly, Rhagoletis pomonella (Walsh) (Casas and Aluja, 1997), the Mexican fruit fly, Anastrepha ludens (Loew) (Robacker et al., 1990), and the Mediterranean fruit fly, Ceratitis capitata (Wiedemann) (Holbrook and Fujimoto, 1969), have also been found to have a different abundance at specific canopy heights, behaving in similar ways. Insect distribution within the canopy could follow a common pattern for frugivorous fruit flies (Balagawi et al., 2012). Therefore they provide a useful focus for modelling.

Although there is evidence showing a significant relationship between host plant architectural characteristics and behaviour of fruit flies, the fundamental scientific questions of how fruit flies optimise their search patterns and limit competition through movement choices and how these movement patterns are affected by plant architecture are still not well understood. There is evidence that underlying rules for generating insect movement behaviour patterns in plant canopies may be simple and generic, based largely on plant architecture and some simple insect behavioural rules (Cribb et al., 2010; Perkins et al., 2010; Perkins et al., 2008; Perkins et al., 2009). If we hypothesize that spatial patterns of insect populations in plant architecture emerge from behaviour of individual insects, IBM can then be used to better simulate the studied system (Vinatier et al., 2011). Thus, IBM will be the approach taken in the current study. The overall objective is to test whether it is possible to predict fruit fly movement and distribution based on hypothesized behaviour and also assess whether such a model can provide insights that may have been missed in past experimental studies, to investigate what level of detail is best used for addressing different types of questions from entomologists, and to show the advantages and disadvantages of both models.

\section{MATERIALS AND METHODS}

\subsection{Model Design}

In the present study, two 3D individual-based models with different levels of detail have been developed to simulate Qfly (Bactrocera tryoni) behaviours and movement patterns on host plants, one with NetLogo (Wilensky, 1999), and the other with L-studio (Karwowski and Prusinkiewicz, 2004; Prusinkiewicz et al., 2000). Each model contains wild female Qflies and a Valencia orange tree with 600 leaves, in order to be consistent with the experiments conducted by Dalby-Ball and Meats (2000). The average leaf area of Valencia orange trees is around $20 \mathrm{~cm}^{2}$ (Yuan et al., 2005). Qflies are assumed to search for a target (e.g. a host fruit) in 
Wang et al., Spatially explicit individual-based modelling of insect-plant interactions: effects of level of detail in Queensland fruit fly models

models (i.e. normal behavior for egg-laying females). The tree is $1 \mathrm{~m}$ in height and the width of tree is $1.5 \mathrm{~m}$. Both models used Qfly behavioural data described in the literature (Dalby-Ball and Meats, 2000). Rules applied to the individual fly were as follows:

- The fly predominantly moves upward by short hops (a limited distance movement between leaves), and moves back into the centre if it is within the edge of the canopy.

- A short hop ends with the fly finding the nearest leaf in a previously selected random direction.

Qflies are aware of the nearby leaves within a specified range. Sensory range for leaves is composed of two modifiable parameters, the detection radius and sensing angle. In this study, we used $80 \mathrm{~cm}$ and 220 degree as detection radius and sensing angle for the sensing sphere (Figure 1). The reason for using these values is that:

- The distance threshold of the sphere of attraction for the apple maggot fly, Rhagoletis pomonella (Walsh), is around $80 \mathrm{~cm}$ in an apple tree canopy (Verdeny-Vilalta et al., 2014). No experimental data are currently available on this topic for Qfly. Since Qfly and apple maggot fly are from the same family, and demonstrate similar behaviours, for these modelling experiments it is assumed that they have somewhat similar distance thresholds for the sensing sphere.

- To model up and downward movement choices, 220 degrees was chosen in order to facilitate observations that Qflies predominantly move upward (Dalby-Ball and Meats, 2000), but still allow for the possibility of a downward move.

The system algorithm applied to both models is shown in Figure 2. At the beginning of the simulation, a tree is created and female Qflies are randomly located in the lower third part of tree. In each 1 minute time step, if there are any leaves within the sensing sphere, the Qfly will move to the nearest leaf in a randomly selected direction. If no leaves are present, the Qfly will hop randomly and then find the nearest leaf on which to land. The number of visits to a leaf will be incremented when a fruit fly arrives. The simulation will stop at 15 minutes. Each movement (resulting in a visit) has been set to an equivalent of $1 \mathrm{~min}$, because the mean time spent by individual wild Qfly observed by Dalby-Ball and Meats (2000) was about 1 min on a leaf. Therefore, the total number of visits in each region will be the total time spent in that region, in minutes.

The number of visits to each leaf and movement trails among leaves can be displayed either numerically or visually in models. As a result, the movement patterns and population distributions will emerge from the behaviours of individual fruit flies. This kind of display provides output for comparisons between runs of the model using different parameter settings for testing, understanding and analysing Qfly movement patterns.

\subsection{Model Description}

\section{NetLogo Model}

The entities in the NetLogo model are wild female Qflies and the spatial units of the tree and ground represented as cubes (patches in NetLogo terminology). One unit distance in the model is equal to $4 \mathrm{~cm}$ in reality. We assume that a green vegetation cube represents two leaves with the average leaf area around $20 \mathrm{~cm}^{2}$. The extent of the model world is $64 \times 64 \times 50$ cubes. The tree has a closed-canopy (cylinder volume - height: $1 \mathrm{~m}$ and width: $1.5 \mathrm{~m}$ ) containing 300 green vegetation cubes (equivalent to 600 leaves in reality). The foliage density of the closed-canopy is approximately 340 leaves per cubic meter. The canopy is considered to consist of lower, middle and upper parts, each approximately one-third the height of the canopy containing 100 green vegetation cubes. Figure 3 visualises the NetLogo model world and a simulation of individual fly movement through a closed-canopy.

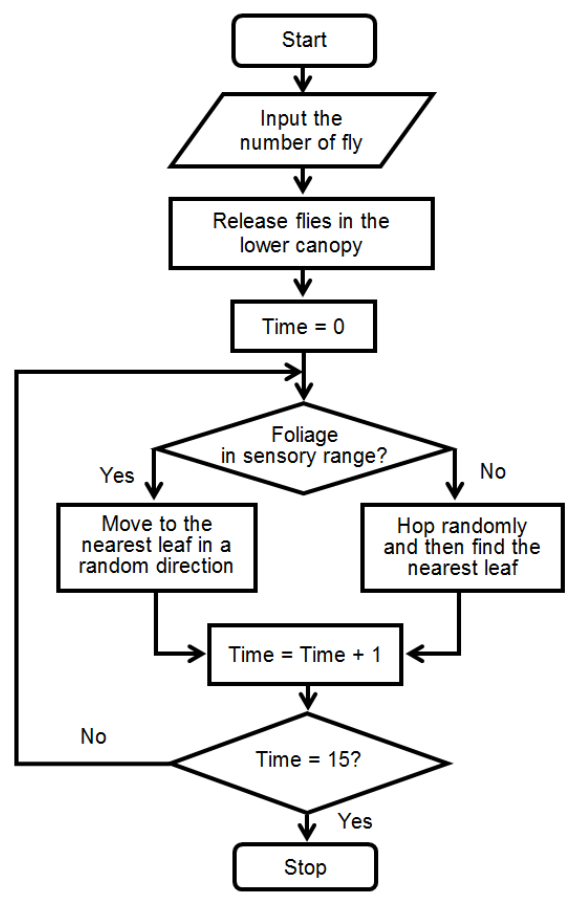

Figure 2. The algorithm flowchart of individualbased Queensland fruit fly model for movement within a simulated tree canopy. 
Wang et al., Spatially explicit individual-based modelling of insect-plant interactions: effects of level of detail in Queensland fruit fly models

\section{L-system Model}

The entities in the L-system model are wild female Qflies and the tree with a more detailed plant architecture. One unit distance in the model is equal to 1 $\mathrm{cm}$ in reality. The tree has a closedcanopy (cylinder volume - height: $1 \mathrm{~m}$ and width: $1.5 \mathrm{~m}$ ) containing 600 leaves. The leaf area of each leaf is 20 $\mathrm{cm}^{2}$. The foliage density of the closedcanopy is approximately 340 leaves per cubic meter. The canopy is considered to consist of lower, middle and upper parts, each approximately one-third the height of the canopy, containing 200 leaves. Figure 4 is a visualisation of the L-system model world and a simulation of individual fly movement through a closed-canopy.

\subsection{Data Analysis}

Statistical analysis of visits/time in the different geometrical zones, e.g. the upper, middle, lower canopy, was undertaken. Each model was run

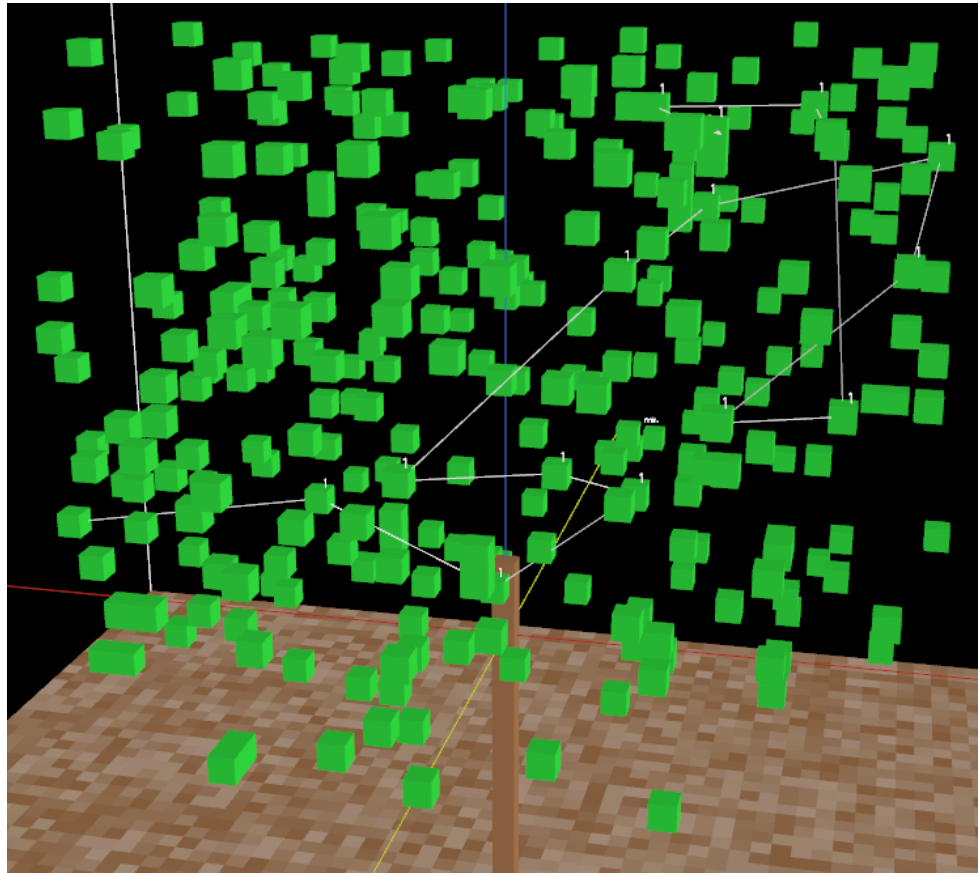

Figure 3. Simulation of individual fly movement through a closedcanopy. The green cubes represent leaves, with number of visits indicated for where the fly has already been. The lines between cubes are hop paths.

(replicated) 50 times and each simulation was set to stop when the time step reached 15 (equivalent to 15 minutes in reality). Model outputs have been checked for normality using the Shapiro-Wilk normality test. The one-way ANOVA followed by the Tukey test were used to test for significant differences between regions. Outcomes of the same geometrical zone from two models were compared using the independent samples t-test. The computational time of the two models was analysed using Mann-Whitney-Wilcoxon test.

\section{RESULTS}

In the NetLogo model, the mean time spent by Qflies in upper region (292.62 mins) was the longest among three regions, followed by the mean time spent in middle region (114.72 mins), and the lower region showed the least time (42.66 mins). These data were found to differ significantly from one another (Figure 5). For the mean time spent in the tree in the L-system model, Qflies visited the upper region (309.86 mins) for almost 3 times the mean time spent in the middle region (106.84 mins), and for almost 7 times the mean time spent in the lower region (33.3 mins). The mean time spent in the middle region (106.84 mins) was 3.2 times as long as the mean time spent in the lower region (33.3 mins). These data were found to differ significantly from one another (Figure 6).

Comparison of the same regions between the two models showed

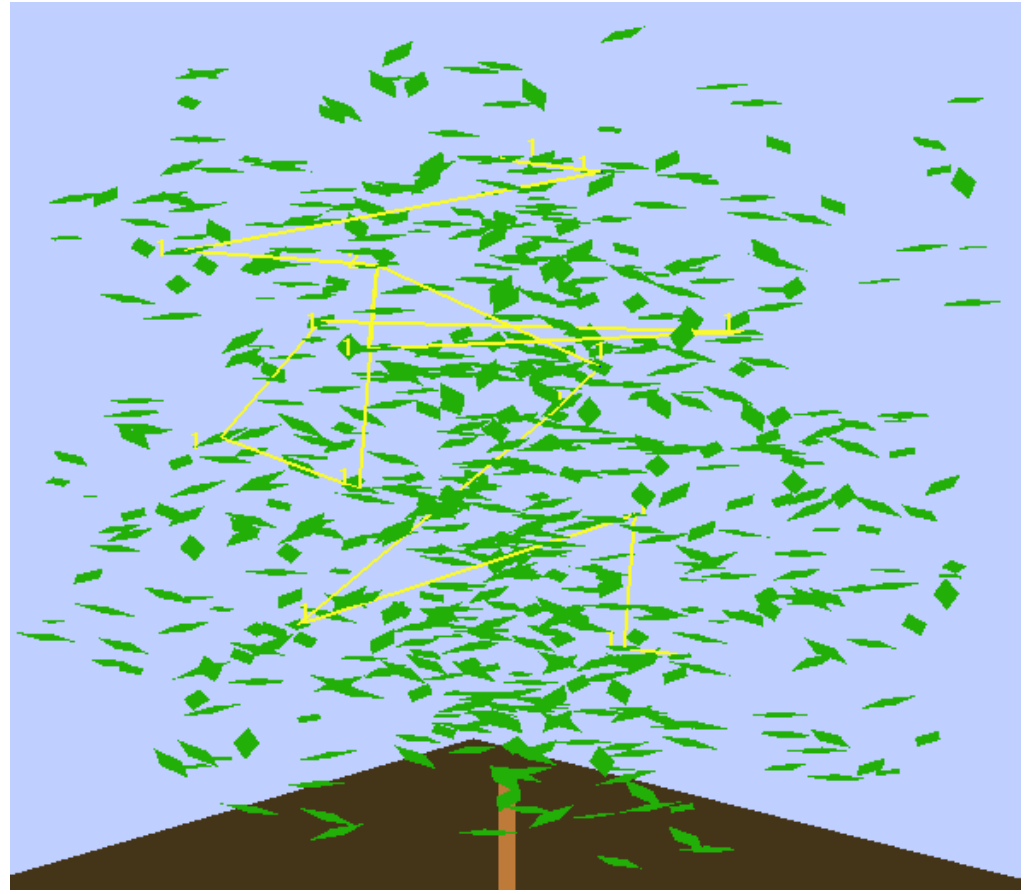

Figure 4. Simulation of individual fly movement through a closedcanopy. Leaves show the number of visits where appropriate. The lines between leaves are hop paths. 
Wang et al., Spatially explicit individual-based modelling of insect-plant interactions: effects of level of detail in Queensland fruit fly models

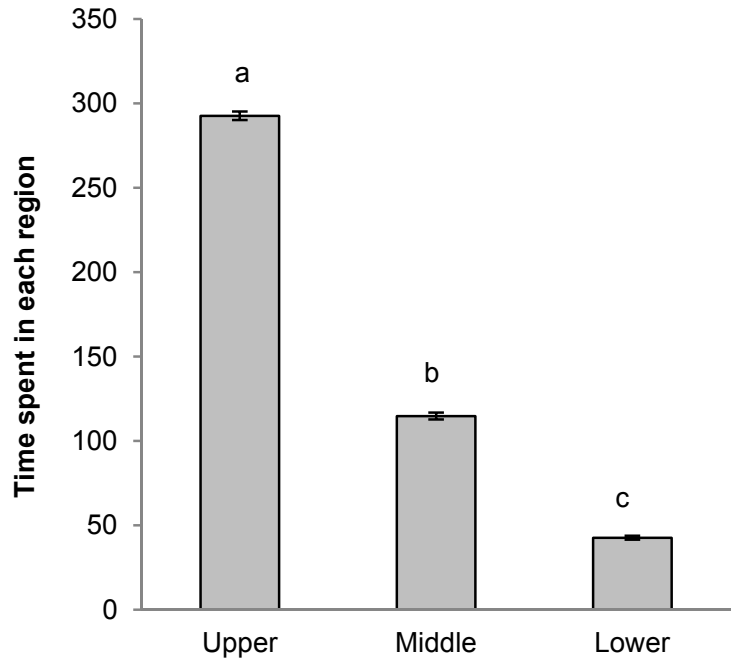

Figure 5. Mean time (min) spent by 30 Qflies in each region in the NetLogo model. Columns with the same letter are not significantly different at $\mathrm{P}=$

0.05 ( $\mathrm{n}=50$ simulations). Upper vs. Middle vs. Lower $($ Mean $=292.62|114.72| 42.66, F=4164.57$,

$$
d f=2, P<0.001) \text {. }
$$

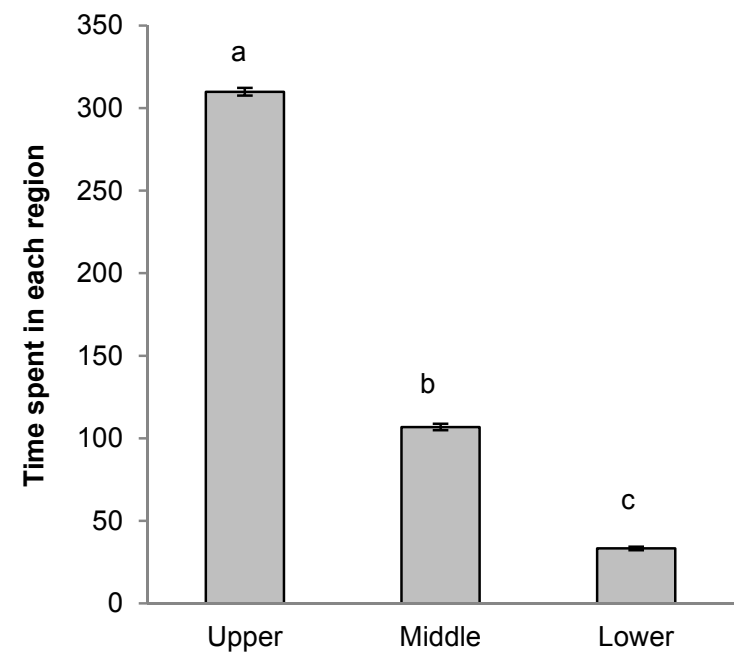

Figure 6. Mean time (min) spent by 30 Qflies in each region in the L-system model. Columns with the same letter are not significantly different at $\mathrm{P}=0.05(\mathrm{n}=50$ simulations). Upper vs. Middle vs. Lower (Mean $=$ $309.86|106.84| 33.3, F=5852.80, d f=2, P<0.001)$.

differences. Flies spent more time in the upper region of the L-system model than of the NetLogo model, whereas in contrast, the time spent in the middle or lower region in the L-system model was less than the time spent in the middle or lower region in the NetLogo model. These data were found to differ significantly from one another (Table 1).

For the computational time, comparison of the two models showed that the computational time of L-system model is much shorter than that of the NetLogo model. These data were found to differ significantly from one another (Table 2).

\section{DISCUSSION AND CONCLUSIONS}

Both models built from published behavioural data with different levels of detail, were found to result in simulated activity consistent with real-world movements of Qflies (as published): the model predicts that most Qflies will be found in the mid to upper canopy. There is general agreement that the abundance

Table 1. The comparison of mean time (min) spent by 30 Qflies in each region of the tree in the two models.

\begin{tabular}{lccccc}
\hline NetLogo vs. L-system & Mean 1 & Mean 2 & $\mathrm{t}$ & $\mathrm{df}$ & $\mathrm{P}$ \\
\hline Upper & 292.62 & 309.86 & -4.99 & 97 & $<\mathbf{0 . 0 0 1}$ \\
Middle & 114.72 & 106.84 & 2.77 & 97 & $\mathbf{0 . 0 0 7}$ \\
Lower & 42.66 & 33.30 & 6.02 & 96 & $<\mathbf{0 . 0 0 1}$ \\
\hline
\end{tabular}

of frugivorous fruit flies at different canopy heights is varied, and the increasing height is generally associated with greater abundance (Balagawi et al., 2012). This argument can be supported by reference to data from the wild tobacco fruit fly, Bactrocera cacuminata (Hering) (Raghu et al., 2004), the Queensland fruit fly, Bactrocera tryoni (Froggatt) (Balagawi et al., 2012), the apple maggot fly, Rhagoletis pomonella (Walsh) (Casas and Aluja, 1997), the Mexican fruit fly, Anastrepha ludens (Loew) (Robacker et al., 1990), and the Mediterranean fruit fly, Ceratitis capitata (Wiedemann) (Holbrook and Fujimoto, 1969). In particular, the model predictions are supported by the findings of Balagawi et al. (2012) in terms of the patterns of abundance of Qflies in different heights of canopy: fewer Qflies are found in the lower canopy compared with the middle and upper canopy. As an applied outcome, Balagawi et al. (2012) proposed that protein bait sprays for Qfly management should be applied as high in the canopy as is mechanically possible for greatest efficacy.

In this study, the NetLogo model is relatively easy to be built, as NetLogo provides a simple yet powerful programming language, built-in user-friendly graphical interfaces, and comprehensive documentation (Railsback et al., 2006). Thus, it can widely be used by a range of users, not only modellers but also biologists, to develop their models quickly. However, computational time can be substantially increased when a large number of

Table 2. The comparison of median computational time (milliseconds) of the two models (Qfly $=30, \mathrm{n}=50$ simulations). 
Wang et al., Spatially explicit individual-based modelling of insect-plant interactions: effects of level of detail in Queensland fruit fly models

agents are applied to the model world. The NetLogo model is less detailed but requires that more time be spent to conduct simulations. On the other hand, computational time of the L-system model is much faster as shown in Table 2, and it provides a realistic tree structure, which may help entomologists gain additional perspectives. Perhaps the disadvantage of the L-system model is that it requires higher level knowledge of computer science, especially computer graphics and programming language. This would limit its learnability and usability.

According to Figure 5 and Figure 6, there is no difference between the two models in terms of spatial population distribution patterns in the plant canopy. Thus, NetLogo can be better used under such circumstances due to the advantage of its learnability and usability. For example, investigating scientific questions like insect spatial population distribution in plant canopies and how different tree architectures affect insect behaviour. This recommendation assumes that those questions do not require a finer spatial resolution.

As Table 1 shows, there is significant difference between the same regions in the two models. Qflies spent more time in the upper region of the L-system model than of the NetLogo model, and correspondingly less in the middle or lower region. The reason for this difference is that the L-system model has a more detailed tree architecture with individual leaves, with gaps between leaves allowing flies to move through what would have been a blocking cube in the NetLogo model. In order to determine the accuracy of these findings, real-world trials will be needed. These findings suggest that the L-system model is better to use to look at how foliage density and foliage position affect fruit fly behaviour. In addition, its faster computational time as seen in Table 2 and finer resolution makes the L-system model more suited to simulate landscape scales such as orchards including multiple trees, than the NetLogo model.

In summary, this study has demonstrated that it is feasible to build an individual-based model from published data to predict fruit fly movement. Such models allow rapid testing of ideas about insect interactions with plant architecture in silico rather than in vivo to generate new perspectives. As a result, model outcomes could be used to inform future experiments. For instance, vase-shaped trees are very common in some Australian orchards (Allan et al., 1993; Campbell et al., 1996). Orchardists modify tree architecture for a number of reasons such as to increase fruit-yield and reduce occurrence of some pests and diseases (Campbell et al., 1996; Simon et al., 2007) but impact on Qfly movement and host-finding success does not appear to have been investigated specifically. The strength of individual-based modelling approach is that predictions can be achieved in a timely fashion from existed simple behavioural data. Those predictions can guide future experiments that will be conducted in the field to investigate host plant architecture and foliage density effects on host-finding. Therefore, time and resources can be better focussed on experimentation that shows the greatest likelihood of success.

\section{ACKNOWLEDGMENTS}

We acknowledge funding for this project from the Plant Biosecurity CRC and Prof. Anthony Clarke and staff at the Queensland University of Technology for support and comment.

\section{REFERENCES}

Allan, P., George, A., Nissen, R., and Rasmussen, T. (1993). Effects of girdling time on growth, yield, and fruit maturity of the low chill peach cultivar flordaprince. Animal Production Science, 33(6), 781-785.

Balagawi, S., Jackson, K., and Clarke, A. (2014). Resting sites, edge effects and dispersion of a polyphagous Bactrocera fruit fly within crops of different architecture. Journal of applied entomology, 138(7), 510-518.

Balagawi, S., Jackson, K., Hamacek, E. L., and Clarke, A. R. (2012). Spatial and temporal foraging patterns of Queensland fruit fly, Bactrocera tryoni (Froggatt) (Diptera: Tephritidae), for protein and implications for management. Australian Journal of Entomology, 51(4), 279-288.

Campbell, J., Nicol, H., and Cullis, B. (1996). Effect of four different canopy shapes on apple yields. Animal Production Science, 36(4), 489-499.

Casas, J., and Aluja, M. (1997). The geometry of search movements of insects in plant canopies. Behavioral Ecology, 8(1), 37-45.

Clarke, A. R., Powell, K. S., Weldon, C. W., and Taylor, P. W. (2011). The ecology of Bactrocera tryoni (Diptera: Tephritidae): what do we know to assist pest management? Annals of Applied Biology, 158(1), 2654.

Cribb, B. W., Hanan, J., Zalucki, M. P., and Perkins, L. E. (2010). Effects of plant micro-environment on movement of Helicoverpa armigera (Hübner) larvae and the relationship to a hierarchy of stimuli. Arthropod-Plant Interactions, 4(3), 165-173.

Dalby-Ball, G., and Meats, A. (2000). Effects of fruit abundance within a tree canopy on the behaviour of wild and cultured Queensland fruit flies, Bactrocera tryoni (Froggatt) (Diptera: Tephritidae). Australian Journal of Entomology, 39(3), 201-207. 
Wang et al., Spatially explicit individual-based modelling of insect-plant interactions: effects of level of detail in Queensland fruit fly models

Di Ventura, B., Lemerle, C., Michalodimitrakis, K., and Serrano, L. (2006). From in vivo to in silico biology and back. Nature, 443(7111), 527-533.

Dyer, A., Dorin, A., Reinhardt, V., Garcia, J., and Rosa, M. (2014). Bee reverse-learning behavior and intracolony differences: simulations based on behavioral experiments reveal benefits of diversity. Ecological Modelling, 277, 119-131.

Fisher, J., and Henzinger, T. A. (2007). Executable cell biology. Nature biotechnology, 25(11), 1239-1249.

Guttal, V., Romanczuk, P., Simpson, S. J., Sword, G. A., and Couzin, I. D. (2012). Cannibalism can drive the evolution of behavioural phase polyphenism in locusts. Ecology letters, 15(10), 1158-1166.

Hanan, J., Prusinkiewicz, P., Zalucki, M., and Skirvin, D. (2002). Simulation of insect movement with respect to plant architecture and morphogenesis. Computers and Electronics in Agriculture, 35(2), 255-269.

Holbrook, F., and Fujimoto, M. (1969). Mediterranean fruit flies and melon flies trapped at various heights with synthetic lures. Journal of Economic Entomology.

Karr, J. R., Sanghvi, J. C., Macklin, D. N., Gutschow, M. V., Jacobs, J. M., Bolival, B., Assad-Garcia, N., Glass, J. I., and Covert, M. W. (2012). A whole-cell computational model predicts phenotype from genotype. Cell, 150(2), 389-401.

Karwowski, R., and Prusinkiewicz, P. (2004). The L-system-based plant-modeling environment L-studio 4.0. In C. Godin, J. Hanan, W. Kurth, A. Lacointe, A. Takenaka, P. Prusinkiewicz, T. DeJong, C. Beveridge, \& B. Andrieu (Eds.), Proceedings of the 4th International Workshop on Functional-Structural Plant Models (pp. 403-405). Montpellier, France: UMR AMAP.

Martin, K. R., Barua, D., Kauffman, A. L., Westrate, L. M., Posner, R. G., Hlavacek, W. S., and MacKeigan, J. P. (2013). Computational model for autophagic vesicle dynamics in single cells. Autophagy, 9(1), 74-92.

Pe'er, G., Saltz, D., Münkemüller, T., Matsinos, Y. G., and Thulke, H. H. (2013). Simple rules for complex landscapes: the case of hilltopping movements and topography. Oikos, 122(10), 1483-1495.

Peck, S. L. (2004). Simulation as experiment: a philosophical reassessment for biological modeling. Trends in ecology \& evolution, 19(10), 530-534.

Perkins, L., Cribb, B., Hanan, J., and Zalucki, M. (2010). The movement and distribution of Helicoverpa armigera (Hübner) larvae on pea plants is affected by egg placement and flowering. Bulletin of entomological research, 100(05), 591-598.

Perkins, L. E., Cribb, B. W., Hanan, J., Glaze, E., Beveridge, C., and Zalucki, M. P. (2008). Where to from here? The mechanisms enabling the movement of first instar caterpillars on whole plants using Helicoverpa armigera (Hübner). Arthropod-Plant Interactions, 2(4), 197-207.

Perkins, L. E., Cribb, B. W., Hanan, J., and Zalucki, M. P. (2009). The role of two plant-derived volatiles in the foraging movement of 1st instar Helicoverpa armigera (Hübner): time to stop and smell the flowers. Arthropod-Plant Interactions, 3(3), 173-179.

Prusinkiewicz, P., Karwowski, R., Měch, R., and Hanan, J. (2000). L-Studio/cpfg: A Software System for Modeling Plants. In M. Nagl, A. Schürr, \& M. Münch (Eds.), Applications of Graph Transformations with Industrial Relevance (Vol. 1779, pp. 457-464): Springer Berlin Heidelberg.

Raghu, S., Drew, R. A., and Clarke, A. R. (2004). Influence of host plant structure and microclimate on the abundance and behavior of a tephritid fly. Journal of Insect Behavior, 17(2), 179-190.

Railsback, S. F., Lytinen, S. L., and Jackson, S. K. (2006). Agent-based simulation platforms: Review and development recommendations. Simulation, 82(9), 609-623.

Robacker, D. C., Moreno, D. S., and Wolfenbarger, D. A. (1990). Effects of trap color, height, and placement around trees on capture of Mexican fruit flies (Diptera: Tephritidae). Journal of Economic Entomology, $83(2), 412-419$.

Simon, S., Sauphanor, B., and Lauri, P.-E. (2007). Control of fruit tree pests through manipulation of tree architecture. Pest Technology, 1(1), 33-37.

Verdeny-Vilalta, O., Aluja, M., and Casas, J. (2014). Relative roles of resource stimulus and vegetation architecture on the paths of flies foraging for fruit. Oikos, 124(3), 337-346.

Vinatier, F., Tixier, P., Duyck, P. F., and Lescourret, F. (2011). Factors and mechanisms explaining spatial heterogeneity: a review of methods for insect populations. Methods in Ecology and Evolution, 2(1), 11-22.

Wilensky, U. (1999). NetLogo. http://ccl.northwestern.edu/netlogo/. Center for Connected Learning and Computer-Based Modeling, Northwestern University, Evanston, IL.

Yuan, R., Alferez, F., Kostenyuk, I., Singh, S., Syvertsen, J. P., and Burns, J. K. (2005). Partial defoliation can decrease average leaf size but has little effect on orange tree growth, fruit yield and juice quality. HortScience, 40(7), 2011-2015.

Zurell, D., Eggers, U., Kaatz, M., Rotics, S., Sapir, N., Wikelski, M., Nathan, R., and Jeltsch, F. (2014). Individual-based modelling of resource competition to predict density-dependent population dynamics: a case study with white storks. Oikos, 124(3), 319-330. 